\title{
Seeing Safety Through the Patient's Eyes
}

Patient harm happens in every healthcare setting: at home in convalescence, in the nursing home at physiotherapy, in an operating room under anaesthesia, in the hospital corridor lying alone on a stretcher, at the walk-in clinic with the paediatrician, in the emergency department awaiting physician assessment. Harm occurs as a result of failures in patient care, rather than from the natural progress of illness or infirmity. Harm may result from wrong or missed diagnosis, scheduling delay, poor hygiene, mistaken identity, unnoticed symptoms, hostile behaviour, device malfunction, confusing instructions, insensitive language and hazardous surroundings.

The trajectory of harm begins with the unexpected experience of harm arising from or associated with the provision of care, including acts of both commission and omission. The initial consequence of harm may be fleeting, temporary or permanent, including death. Harm also may not cease even when the cause is halted. The patient may experience harm during the episode of care when the failure occurred, or later, after some time has passed. Harm as it is first endured may evolve, transform and spread. Over time, untreated harm may cause further damage to the initial victim, both temporary and permanent, and to many others. (Canfield 2013)

Consider these reflections on patient harm written by Carolyn Canfield whose husband's care was very poorly managed at the end of his life. This is a description of harm written from the patient's side and in several respects it is strikingly different from the accounts of incidents and adverse events described by healthcare professionals. Three things in particular stand out:

- First, harm is conceived very broadly encompassing both serious disruption of treatment and lesser events that are more distressing than injurious.

- Second, harm for a patient includes serious failures to provide appropriate treatment as well as harm that occurs over and above the treatment provided. Very poor quality care is therefore seen as harmful and included within patient safety.

- Third, and perhaps most important, harm is seen not in terms of incidents but as a trajectory within a person's life. Both the genesis and consequences of harm occur over time and the timescales are very much longer than those normally considered. Incidents are simply those aspects of harm that are observed by healthcare professionals and, while important, they are a necessarily incomplete vision.

C. Vincent, R. Amalberti, Safer Healthcare: Strategies for the Real World,

DOI 10.1007/978-3-319-25559-0_4 
We are now moving to a rather different vision of patient safety. Our previous vision might reasonably be described as one of generally high quality healthcare punctuated by occasional safety incidents and adverse events. We now recast patient safety as the examination of serious failures and harm along the patient journey which must of course be seen in the context of the benefits of the healthcare received. This requires us to view both benefit and harm from the perspective of the patient, not because this is ideologically or politically correct, but because this is the reality we need to capture.

\section{What Do We Mean by Harm?}

Patient safety initially focused on rare, often tragic, events. However, as safety was more systematically studied it became clear that the frequency of error and harm were much greater than previously realised and that the safety of all patients needed to be addressed. Most patients are vulnerable to some degree to infections, adverse drug events, falls, and the complications of surgery and other treatments. Patients who are older, frailer or have several conditions may experience the adverse effects of over-treatment, polypharmacy and other problems such as delirium, dehydration or malnutrition. In addition, patients may also suffer harm from rare and perhaps unforeseeable events, stemming from new treatments, new equipment or rare combinations of problems that could not easily have been foreseen. If we want to assess harm from healthcare then we have to consider all these kinds of events.

Harm can be defined in various ways and there is no absolute border, particularly as the perimeter of patient safety is constantly expanding as we have discussed. Some types of events, such as a drug overdose with consequences for the patient, can be clearly described as a harmful event caused by healthcare. Harm that results from a failure of treatment is more difficult. For instance if a patient was not given appropriate prophylactic medication and then suffered a thromboembolism the harm, or at least the causation, is not so clear cut. With diagnostic delay, the notion of harm is more difficult still. Increasingly though failures to recognise deterioration and failure to institute treatment are being described as patient safety issues (Brady et al. 2013). Whether or not we would describe all these events as harmful we can all agree that they are undesirable and represent serious failures in the care of the patients concerned (Box 4.1).

\section{Box 4.1 Examples of Types of Harm in Healthcare}

General harm from healthcare

Hospital-acquired infections, falls, delirium and dehydration are examples of problems that can affect any patient with a serious illness.

Treatment-specific harm

Harm that is associated with a specific treatment or the management of a particular disease which may or may not be preventable. This would include adverse drug events, surgical complications, wrong site surgery and the adverse effects of chemotherapy. 


\section{Harm due to over-treatment}

Patients may also be harmed from being given too much treatment, either through error (for instance a drug overdose) or from well-intended but excessive intervention. For example, excessive use of sedatives increases the risk of falls; people near the end of life may receive treatments that are painful and of no benefit to them.

Harm due to failure to provide appropriate treatment

Many patients fail to receive standard evidence-based care and for some this means their disease progresses more rapidly than it might. Examples include failure to provide rapid thrombolytic treatment for stroke, failure to provide treatment for myocardial infarction, and failure to give prophylactic antibiotics before surgery.

Harm resulting from delayed or inadequate diagnosis

Some harm results because the patient's illness is either not recognised or is diagnosed incorrectly. For example, a patient may be misdiagnosed by their primary care physician, who fails to refer them; the cancer advances and the outcome may be poorer.

Psychological harm and feeling unsafe

Instances of unkindness can linger in the memory of vulnerable people and affect how they approach future encounters with healthcare professionals. Awareness of unsafe care may have wider consequences if it leads to a loss of trust. For instance, people may be unwilling to have vaccinations, give blood or donate organs.

Adapted from Vincent et al. (2013)

Reflecting on the many ways in which healthcare can fail or harm patients it is clear that much of it is insidious, develops slowly and, if not addressed, may result in a crisis involving admission to hospital or other urgent treatment. A frail person in hospital who gradually becomes delirious receives care that is sub-standard to the point of being harmful but this cannot really be captured by thinking in terms of errors or incidents. When we consider care outside hospital the concept of 'an incident' breaks down even further. Consider, for instance, a patient who reacts adversely to prescribed anti-depressant medication over a period of several months culminating in an admission to hospital. We know that adverse drug reactions are implicated in about $5 \%$ of hospital admissions (Winterstein et al. 2002; Stausberg et al. 2011) but the harm preceding these events has a timescale of months. Moreover harm of this kind cannot be seen in terms of 'error' at least not an error on any specific occasion.

\section{Safety and Quality of Care from the Patient's Perspective}

When we view our care as patients we see the course of our disease and the care we receive over time and in the context of our lives. Of course there are episodes of care but we assess our experience and the impact of healthcare in terms of the totality of 

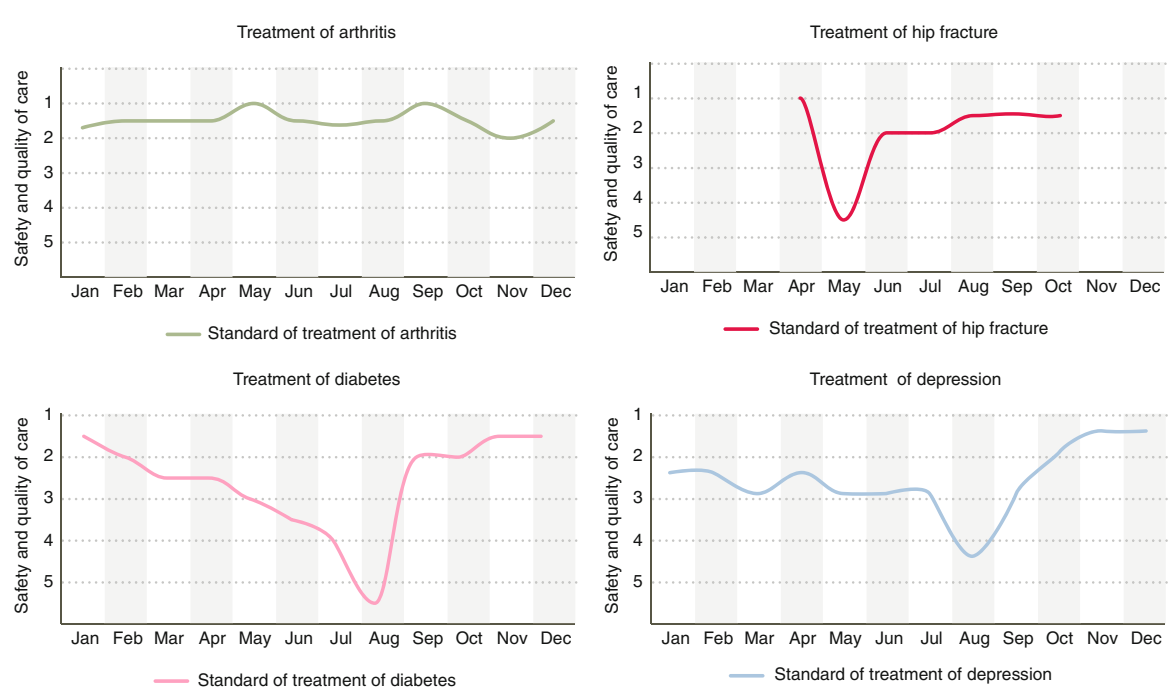

Fig. 4.1 Four patient journeys

our treatment and the overall balance of benefit and harm. How can we represent this in terms of the safety and quality of care and the various scenarios that we might wish to encompass?

Consider the four patient journeys represented in Fig. 4.1. In each case the graph provides a simple representation of the quality of care each patient receives over 1 year for the treatment of different conditions. The horizontal axis represents time and the vertical axis shows the standard of care provided (not the health of the patient or severity of their condition). The five levels discussed in Chap. 2 are shown on the left with good care shown at the top of the graphs with deteriorating or dangerous care shown by declining levels.

- The first person is receiving long-term treatment for osteoarthritis. Both treatment and monitoring are of a high standard, consistent over time and the overall quality of care is excellent.

- The second person sustains a fracture of the hip in April. Initial treatment is excellent, with prompt admission to hospital and rapid and effective surgery. However, in the post-operative period the patient develops a serious wound infection which is not immediately recognised by the nurse visiting at home. The infection worsens, a second admission to hospital is required but the infection is effectively treated and recovery is then uneventful. Overall quality of care is good, with one serious lapse during May.

- The third person is being treated for diabetes, initially effectively. However from the beginning of the year care deteriorates in that monitoring is not effective and 


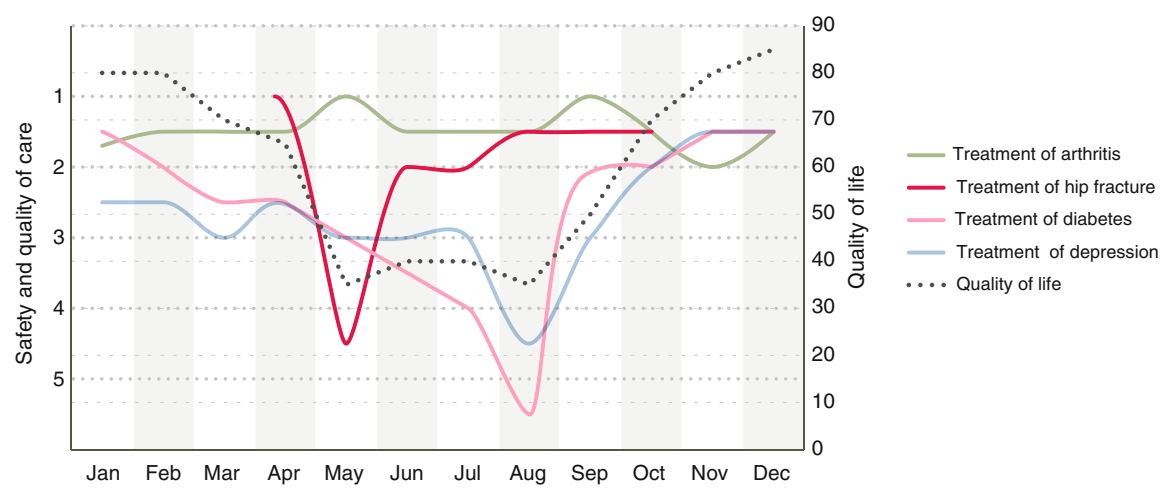

Fig. 4.2 Varying standards of care over time

becomes progressively more sporadic, resulting in admission to hospital with potentially life threatening complications in August. Hospital care is excellent however and the patient makes a good recovery.

- The fourth person is receiving partially effective treatment for depression. Monitoring is infrequent, treatment is not optimal but there are no major crises during the year. Quality of care hovers around our level 3 - poor, but still reasonably safe.

These four scenarios are relatively straightforward and, in many healthcare settings, the patient's care and progress could be monitored to some extent. We could assess the outcome of the hip replacement, record the post-operative infection and monitor the frequency of treatment for arthritis, diabetes and depression in primary care. However, we should now consider a different but more realistic scenario that more truly represents the balance of benefit and harm that we ideally wish to capture, understand and influence. This is the person who suffers from and receives treatment for a number of conditions at the same time.

Consider a person who suffers from arthritis, diabetes and depression and who sustains a hip fracture during the year. Figure 4.2 shows the four individual graphs combined for this patient with an additional second axis to give an assessment of the overall quality of life for this patient during this year of tribulation. As before the care for arthritis remains of high quality throughout the year, the care of the hip fracture generally very good but interrupted by an initially unrecognised post-operative infection. Treatment of diabetes remains problematic and we might suspect, from looking at the decline in quality of care after April, that the hospital admission and subsequent infections may have disrupted the usual monitoring. We can also very clearly see how complex healthcare is from the perspective of the patient and that very few of the healthcare professionals involved are likely to have a sense of the impact of good and poor healthcare on this person's life. 


\section{Safety Through the Patient's Eyes}

In this chapter we have conceptualised safety in the context of a patient's healthcare journey showing good quality care but also encompassing a number of types of serious failure and harm. The implications of this way of approaching safety will be explored in detail in the second section of the book but it will be useful to indicate some general directions here.

\section{The Patient Potentially Has the Most Complete Picture}

The most obvious point to emerge from studying treatment over time is that the patient is, even more than in hospital, a privileged witness of events. Patient reported outcome measures are of course already a high priority, but we clearly need to begin to find ways of tracking patient experience of healthcare over time and integrating this information with available clinical information. This is easy to say but likely to be a task of considerable difficulty.

\section{The Healthcare professional's View Is Necessarily Incomplete}

Each healthcare professional involved with a patient will only have a partial view of the patient journey. Even within hospital, whether notes are electronic or paper, it can be difficult to understand the trajectory of patient care. The problem is even more acute outside hospital. A good general practitioner or family doctor is best positioned to understand the full patient journey, but we will need to develop methods of representing the full perspective of care that can be shared across different settings.

\section{The Resources of the Patient and Family Are Critical to Safe Care}

Increasingly patients and families are managing the complex work of coordinating their care. The formal assessment of these resources, financial, emotional and practical will become essential to the coordination of care and the idea of the patient as part of the healthcare team will move from being an aspiration to a necessity. This can certainly bring benefits in terms of patient engagement and patient empowerment but also carries risks as patients shoulder the burden of organising and 
delivering care and the locus of medical error moves from professionals to patients and families.

\section{Coordination of Care Is a Major Safety Issue}

Patients with multiple problems already have multiple professionals involved in their care and face major challenges in coordinating their own care. Poor communication across different settings is frequently implicated in studies of adverse events in hospital and in inquiries into major care failures in the community. Safety interventions in these settings may be less a matter of care bundles and more concerned with wider organisational interventions to ensure rapid response to crises and coordination between agencies.

\section{Rethinking Patient Safety}

At the beginning of this chapter we argued that the way we currently view patient safety assumes a generally high quality of healthcare punctuated by occasional safety incidents and adverse events. Increasingly we see this as a vision of safety from the perspective of healthcare professionals. This is a sincere vision in that professionals naturally assume that for the most part they are giving good care though they know that there are occasional lapses.

In contrast we have expanded our view of harm and recast patient safety as the examination of the totality of serious failures and harm within the patient journey which must necessarily be set against the benefits of the healthcare received. This is a vision of safety from the perspective of the patient, carer and family.

We believe that future progress in safety depends on conceptualizing safety in this broader manner and linking our understanding of safety with the wider ambitions and purposes of the healthcare system. This means viewing the risks and benefits of treatments over a longer timescale across different contexts and critically within the realities of a fragmented system with multiple vulnerabilities. This will require moving from a focus on specific errors and incidents to examining the origins of more fundamental failures of care such as avoidable hospitalisation due to undetected deterioration in a long term condition. The longer term aim both for patients and for patient safety is to consider how risk and harm can be minimised along the patient journey in pursuit of the optimum benefits from healthcare. In the following chapters we develop these ideas in more detail and consider how this ambitious, but we believe necessary, programme might be undertaken. 


\section{Key Points}

- Patients have a different view of harm to professionals. Harm is conceived very broadly encompassing both serious disruption of treatment and lesser events that are more distressing than injurious.

- Harm for a patient includes serious failures to provide appropriate treatment as well as harm that occurs over and above the treatment provided. Both benefit and harm are seen not in terms of incidents but as a trajectory within a person's life.

- Many patient-identified events are not captured by the incident reporting system or recorded in the medical record.

- We propose that patient safety should focus on the totality of harm within the patient journey which must necessarily be set against the benefits of the healthcare received. This is a vision of safety from the perspective of the patient, carer and family.

- Patients and families will increasingly need to be actively involved in promoting safety. This can certainly bring benefits in terms of patient engagement and patient empowerment but also carries risks as patients shoulder the burden of organising and delivering care and the locus of medical error moves from professionals to patients and families.

- We need to view the risks and benefits of treatments over a longer timescale, across different contexts and within the realities of a fragmented system with multiple vulnerabilities. This will require moving from a focus on specific incidents to examining more fundamental longer term failures such as avoidable hospitalisation due to undetected deterioration in a chronic condition.

- We believe that future progress in safety depends on conceptualizing safety in this broader manner and linking our understanding of safety with the wider ambitions and purposes of the healthcare system.

Open Access This chapter is distributed under the terms of the Creative Commons Attribution Noncommercial License, which permits any noncommercial use, distribution, and reproduction in any medium, provided the original author(s) and source are credited.

\section{References}

Brady PW, Muething S, Kotagal U, Ashby M, Gallagher R, Hall D, Goodfriend M, White C, Bracke TM, DeCastro V, Geiser M, Simon J, Tucker KM, Olivea J, Conway PH, Wheeler DS (2013) Improving situation awareness to reduce unrecognized clinical deterioration and serious safety events. Pediatrics 131(1):e298-e308

Canfield C (2013) It's a matter of trust: a framework for patient harm. Unpublished manuscript.

Stausberg J, Halim A, Färber R (2011) Concordance and robustness of quality indicator sets for hospitals: an analysis of routine data. BMC Health Serv Res 11(1):106. doi:10.1186/1472-6963-11-106

Vincent C, Burnett S, Carthey J (2013) The measurement and monitoring of safety. Health Foundation, London

Winterstein AG, Sauer BC, Hepler CD, Poole C (2002) Preventable drug-related hospital admissions. Ann Pharmacother 36(7-8):1238-1248 